\title{
Ejaculatio praecox: Informationsstand sehr heterogen
}

_ Urologen wissen recht gut über Ursachen und Therapie bei Ejaculatio praecox Bescheid. Die Prävalenz der Erkrankung wird jedoch unterschätzt, so das Ergebnis einer Umfage von Springer Medizin bei Urologen zum Thema vorzeitiger Samenerguss. Jeder zweite Teilnehmer (50\%) nannte als häufigste Ursache korrekt eine angeborene Störung im zentralen Serotonin-TransporterSystem. Bei einer Umfrage unter Allgemeinmedizinern gab dies nur jeder Dritte (34\%) an. $40 \%$ der Urologen nannten eine psychische Belastungssituation als Hauptursache, im Vergleich zu 57\% der Allgemeinmediziner. Die Prävalenz der Erkrankung wurde von beiden Arztgruppen im Vergleich zu Angaben aus der Literatur unterschätzt: Fast drei Viertel (74\%) der Allgemeinmediziner und zwei Drittel (68\%) der Urologen nannten eine Prävalenz von unter $20 \%$, jeder dritte sogar von unter $10 \%$. Nur jeder fünfte in beiden Gruppen gab die Prävalenz mit $21-30 \%$ an.
Bei der Frage nach den Therapieempfehlungen zeigte sich, dass die 50 teilnehmenden Urologen vertrauter mit der Erkrankung sind als die 210 Allgemeinmediziner. Drei Viertel (76\%) der Urologen setzen demnach auf eine medikamentöse Therapie, zum Beispiel auf die Bedarfstherapie mit dem kurzwirksamen SSRI Dapoxetin (Prili$\mathrm{gy}^{\circledast}$ ). Bei den Allgemeinmedizinern waren dies nur $59 \%$. Ähnlich stark ausgeprägt war der Rat zur Verhaltenstherapie wie "StoppStart-Technik" oder "Squeeze-Technik" mit $62 \%$ bei den Urologen und $54 \%$ bei den Allgemeinmedizinern. Lokale Therapien wurden von jedem zweiten (52\%) Urologen und jedem dritten (30\%) der Allgemeinärzte empfohlen, etwa lidocainhaltige Cremes. Mehrfachnennungen waren hier möglich. Und kommen die Teilnehmer mit ihrem Latein ans Ende, würden $22 \%$ der Urologen an einen Sexualtherapeuten überweisen und $30 \%$ der Allgemeinärzte an einen Urologen.
Bei den Kriterien für die Diagnose einer Ejaculatio praecox hatten die Urologen erneut die Nase vorn: Die intravaginale Ejakulationslatenzzeit (IELT) wurde von $58 \%$ der Urologen, aber nur von $34 \%$ der Allgemeinärzte genannt. Die IELT ist ein Kriterium der Definition der primären Ejaculatio praecox. Die Unfähigkeit, die Ejakulation zu verzögern oder zu kontrollieren sowie die psychische Belastung für den Patienten oder den Partner wurde von beiden Gruppen ähnlich häufig genannt.

Weitere Ergebnisse: $44 \%$ der Urologen gaben an, dass die Sexualanamnese in ihrer Praxis regelmäßiger Bestandteil des Patientengesprächs sei. $82 \%$ der Urologen betreuen bis zehn Patienten pro Quartal, die eine Ejaculatio praecox haben. $44 \%$ betreuen sogar fünf bis zehn Patienten mit der Erkrankung. Bei den Allgemeinmedizinern waren das $46 \%$ und $27 \%$.

Dr. Michael Hubert

Umfragen von Springer Medizin, Neu-Isenburg, mit Unterstützung von Berlin-Chemie, Berlin

\section{Erster quantitativer Blasenkrebs-Marker vorgestellt}

_ Risikopersonen für Blasenkrebs können einfach und kostengünstig mit Urin-basierten Proteinmarkern für die patientennahe Diagnostik untersucht werden. Mit UBC Rapid steht jetzt erstmals ein Point-of-Care (POC) Test für die quantitative Auswertung

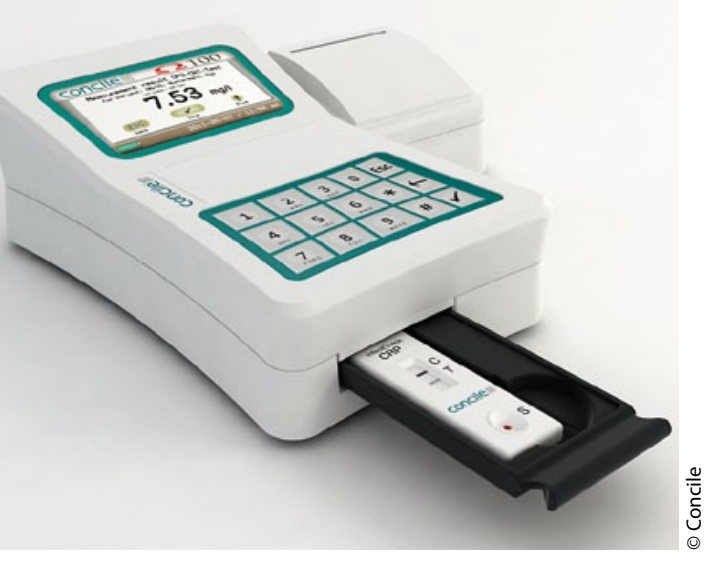

Das Messgerät concile ${ }^{\circledR} \Omega 100$. zur Verfügung. Der UBC Rapid Test kann auf einem kleinen, mobilen POC-Messgerät quantifiziert werden. Dazu wird eine verdünnte Urinprobe des Patienten in das Probenfenster einer Testkassette pipettiert. Die Farbintensität der Testlinie wird in dem Messgerät concile ${ }^{\circledast} \Omega 100$ automatisch mit einem CCD-Sensor anhand einer Kalibrationskurve ausgewertet. Das Gerät ist für die einfache patientennahe Diagnostik vieler verschiedener Marker geeignet. Nach zehn Minuten zeigt es die UBC-Konzentration im Urin in $\mu \mathrm{g} / \mathrm{l}$ an.

Der quantitative UBC Rapid Test zeigte in einer Pilotstudie der Universität Tübingen mit $62,3 \%$ eine signifikant bessere Sensitivität als NMP22, für das mit einer Sensitivität von $16,3 \%$ ein im Vergleich zu früheren Studien deutlich niedrigerer Wert festgestellt wurde. Die quantitative Auswertung des Blasenkrebs-Markers UBC ermöglicht zudem eine bessere Risikobeurteilung als bisherige Schnelltests, so Tilman Todenhöfer von der
Universitätsklinik Tübingen, wo diese Studie durchgeführt wurde. An der Studie nahmen 198 Patienten teil, bei denen ein Verdacht auf ein Harnblasenkarzinom bestand oder die zur Rezidivkontrolle kamen. Bei 39 Patienten lag ein Ersttumor vor, bei 22 weiteren Patienten ein Rezidiv.

UBC Rapid detektiert die Tumor-assoziierten Zytokeratin-Fragmente 8 und 18. Der LateralFlow-Test kann wie NMP22 und BTA stat auch als qualitativer Test mit dem bloßen Auge abgelesen werden. Die POC-Tests zur Diagnose von Blasenkrebs variieren hinsichtlich ihrer Sensitivität, Spezifität und der Anfälligkeit gegenüber Störfaktoren wie Hämaturie und Harnwegsinfektionen. So zeigt der BTA stat Test häufig ein falsch positives Ergebnis bei Blutbeimengungen im Urin und seine Aussagekraft ist bei Hämaturie, dem Leitsymptom des Harnblasenkarzinoms, limitiert. Todenhöfer wies darauf hin, dass bisher noch kein Marker die Zystoskopie ersetzen kann. Die POC-Tests seien jedoch einfach und schnell durchführbar.

Nach Informationen von

Concile, Freiburg 